\title{
A Case for Diagnosis
}

\section{A NEW SYNDROME?}

\author{
ZAIDA M. HALL,* D.M., M.R.C.P. \\ Lately Senior Registrar, Paddington and \\ Kensington Chest Clinic, London
}

BY

AND

\author{
R. R. WILSON, M.Đ. \\ Consultant Morbid Anatomist, Paddington \\ General and St. Charles' Hospitals, London
}

[Wrth Special Plate]

This report describes the case of a man who has had for at least five years, and probably twelve years, numerous focal sclerosing lesions in the lungs and a few in the bones. He has been in excellent health throughout, and would never have been investigated but for a chance $x$-ray examination. No diagnosis has yet been made, in spite of repeated radiographs, three successful biopsies, and other investigations. The case presents two outstanding problems: firstly, the relationship between the lung and bone lesions, and, secondly, the nature of the disease.

We hope that this report may be followed by others so that in time the condition may be better understood.

\section{Case History}

The patient, a man then aged 42 , had a routine mass $x$-ray examination in 1956 , because the unit happened to be in his locality. He had no symptoms. The radiograph showed multiple foci of low density and blurred outline. The patient was referred to the local chest clinic and admitted to St. Charles' Hospital for investigation.

Past History.-In 1942-3 when in North Africa he had an attack of dysentery. Otherwise he had never been out of England. However, in 1945 he had a short attack of benign tertian malaria. Apart from these illnesses he had always had excellent health. In 1949 he had a routine mass $x$-ray examination which was reported as normal. On re-scrutiny, however, three small round shadows were found in the right lower zone, and other less obvious shadows, scattered in both lungs, were also seen. Having previously been a messenger, a footman, a funfair attendant, a cook, and an electro-plater, the patient became a dustman in 1950 .

Examination.-He was a lean but fairly muscular man of medium build and healthy appearance. Respiratory system : no clubbing or cyanosis; chest movements normal; percussion and auscultation normal; diaphragm moved normally on screening. Cardiovascular system: heart rate and rhythm normal ; no murmurs ; B.P. 120/80. Alimentary system: liver not palpable; rectal examination normal; no other abnormality found. Haemopoietic system: spleen not palpable; a few small glands in axillae and groins; no clinical anaemia. Urogenital system: kidneys not palpable; genitalia normal. Nervous system: no abnormality found; fundi normal. Osseous system: no bony tenderness.

Investigations.-Mantoux $1 / 10,000$ negative, $1 / 1,000$ positive. Histoplasmin skin test negative $(1 / 100)$. Histoplasmin complement-fixation test negative. $\mathrm{Hb}, 85 \%$. W.B.C. normal. E.S.R. $5 \mathrm{~mm}$./hour. Urine normal. Chromatography showed no abnormal amino-acids. Plasma protein, 7.8 g. $/ 100 \mathrm{ml}$. (albumin $4.2 \mathrm{~g}$., globulin $3.5 \mathrm{~g}$.); A:G ratio 1.2:1. Serum acid phosphatase, 2.3 units; alkaline phosphatase, $4.7 \mathrm{~K}$-A units ; inorganic phosphorus, $4.3 \mathrm{mg} . / 100 \mathrm{ml}$; ; calcium, $10.5 \mathrm{mg} . / 100 \mathrm{ml}$. W.R. and G.C.F.T. negative. Repeated sputum and laryngeal swab cultures: no tubercle bacilli grown, no fungi grown. Scalene node biopsy: only fatty tissue obtained. Radioactive iodine studies: no evidence of increased uptake by lungs or lumbar spine after an oral dose of $50 \mathrm{mg}$. of ${ }^{131} \mathrm{I}$. Bronchoscopy (1959): no abnormality. Lung-function tests (1960): Ventilatory function is good but a very slight degree of airway obstruction is present. Diffusion is slightly

*Now at 13 Kingsgate Street, Winchester. reduced below the normal limits at rest but rises adequately with exercise. The defects do not suggest such relatively common pictures as are found in emphysema and interstitial pulmonary fibrosis, and in fact are virtually within normal limits for his age.

\section{Lungs.-There was generalized nodular shadowing} throughout both lungs. The nodules varied from 3 to $14 \mathrm{~mm}$. in diameter, were of low density, and had soft blurred outlines. There was a little calcification in some of them (confirmed by tomography). There was no hilar calcification. (See Figs. 1 to 4 .)

Bones.-In the skeleton were six round translucent zones, 14 to $20 \mathrm{~mm}$. in diameter, and several patches of sclerosis. Each translucent area had a central nucleus of denser opacity about $2 \mathrm{~mm}$. across, and also a complete or partial dense peripheral rim about $1 \mathrm{~mm}$. thick. Of the six translucent areas, three were in the pelvis, one in the right femur, one in the third lumbar vertebra, and one in the right second rib.

One lesion was in the left side of the sacrum overlying the sacro-iliac joint. There was a patch of sclerosis over each sacro-iliac joint.

Fig. 5 shows a second lesion, in the right ilium near the brim of the pelvis, surrounded on its upper and medial aspect by an area of sclerosis at least $15 \mathrm{~mm}$. thick. There was no opaque rim on the infero-lateral border of this translucent area.

A third lesion lay just below the right iliac crest. Beyond the narrow border of denser opacity it was. surrounded by an area of sclerosis $20 \mathrm{~mm}$. thick.

A fourth round translucent zone (Fig. 6) was in the right femur below the greater trochanter, the convex outer edge extending $2 \mathrm{~mm}$. over the margin of the femur and being demarcated superficially by a hair-line of densebone. There was no sclerosis above this lesion, but only below and medially, extending for $2-3 \mathrm{~mm}$. One centimetre below the lesion was a rim of periosteal bone formation $3 \mathrm{~mm}$. thick, extending for $3 \mathrm{~cm}$. and showing. longitudinal and vertical striations.

The body of the third lumbar vertebra was uniformly sclerosed except for a fifth central translucent area $2 \mathrm{~cm}$. in diameter.

The sixth lesion, in the inferior border of the anterior end of the right second rib, was seen as a semicircular bite out of the rib, with a margin of sclerosis less than $1 \mathrm{~mm}$. thick along the curved upper border. This margin did not extend below to complete the outline of the lesion.

In addition to the lesions in the lungs and bone there was below the left diaphragm a more calcified ring shadow 10 by $13 \mathrm{~mm}$., with a dense central nucleus. This lesion lay posteriorly in the abdomen and was mobile: it was probably in the mesentery.

\section{X-ray Examination in 1960}

The chest $x$-ray picture has gradually changed, the blurred shadows of 1956 having been replaced by more numerous discrete round shadows. It is probable that the ill-defined shadows of 1956 were due to the superimposition of lesions. now shown by their sharper outlines to be separate. There: 
ZAIDA M. HALL AND R. R. WILSON: A NEW SYNDROME?

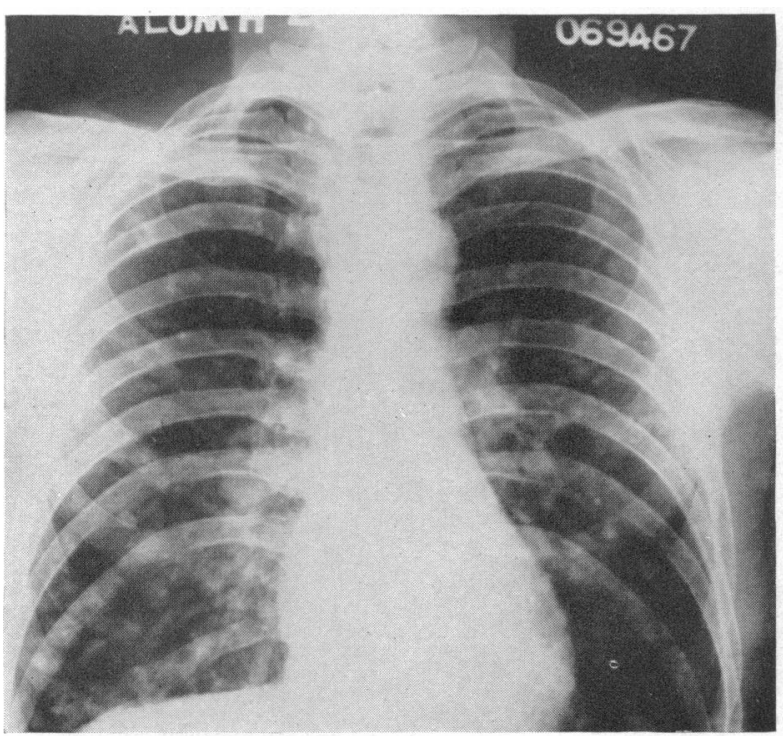

FIG. 1

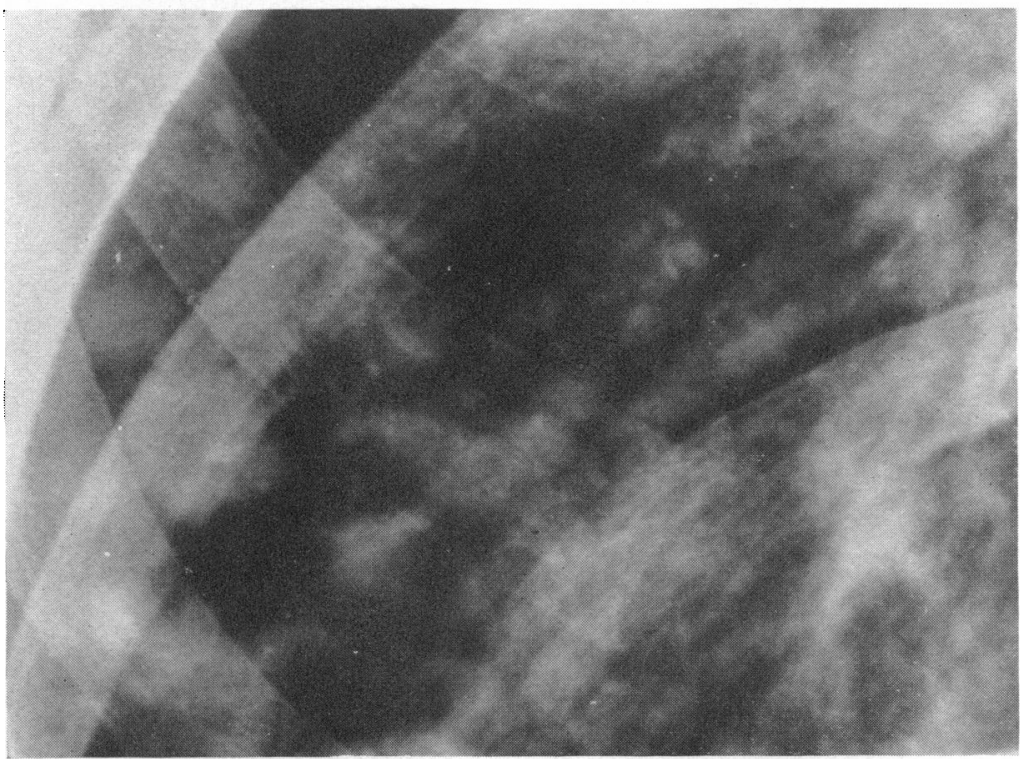

FIG. 3

FIGS. 3 and 4.--Radiographs of the right costophrenic angle taken in 1956 and 1960 respectively.

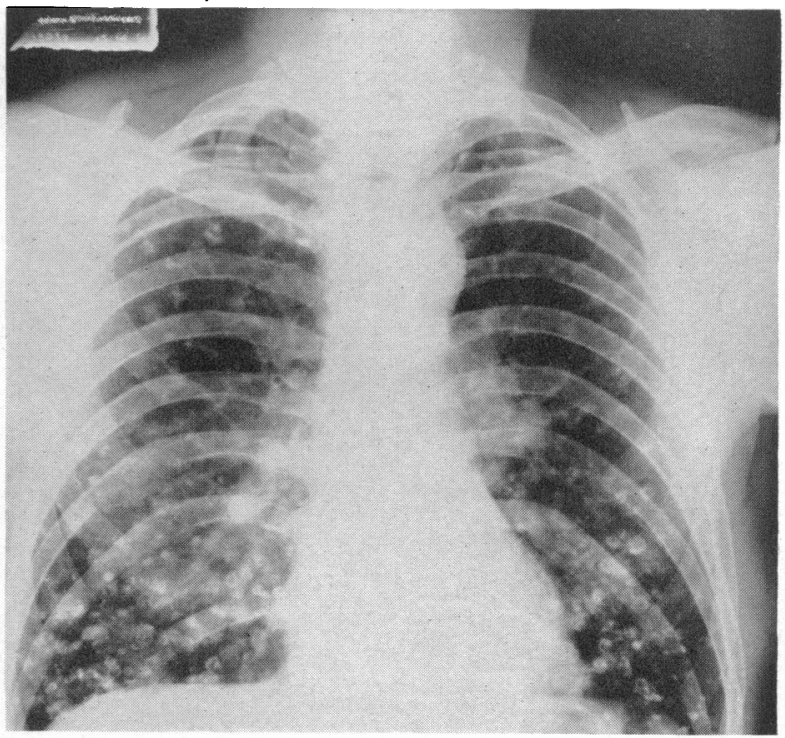

FIG. 2

FIGs. 1 and 2.- Chest radiographs taken in 1956 and 1960 respectively.

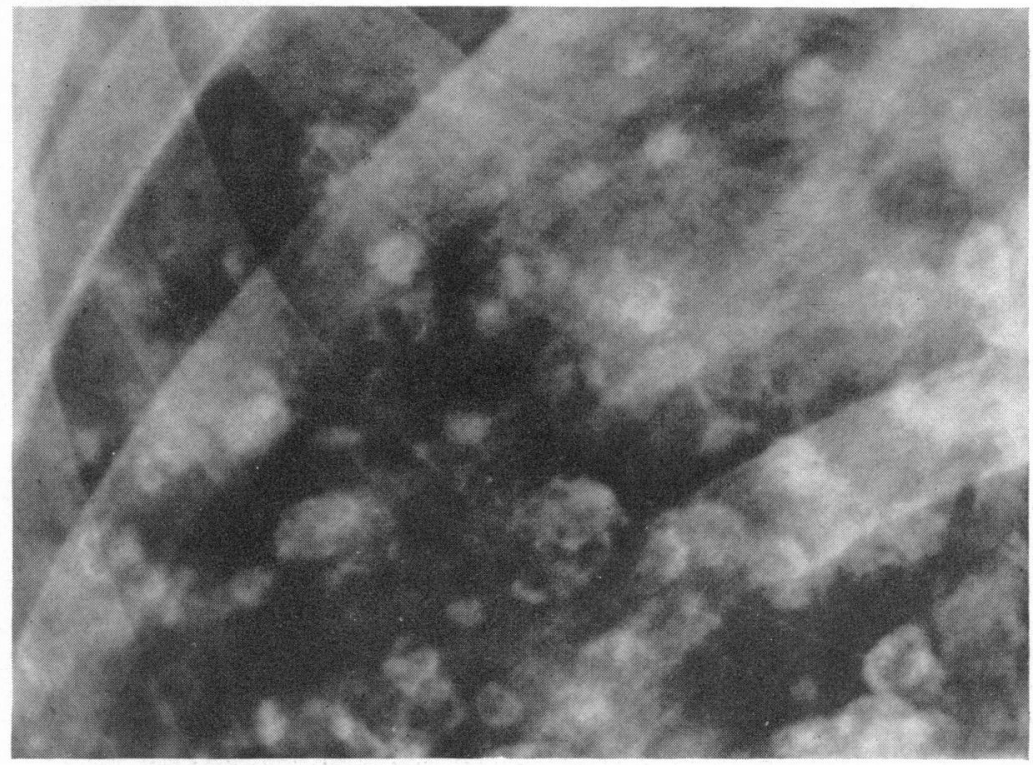

FIG. 4 


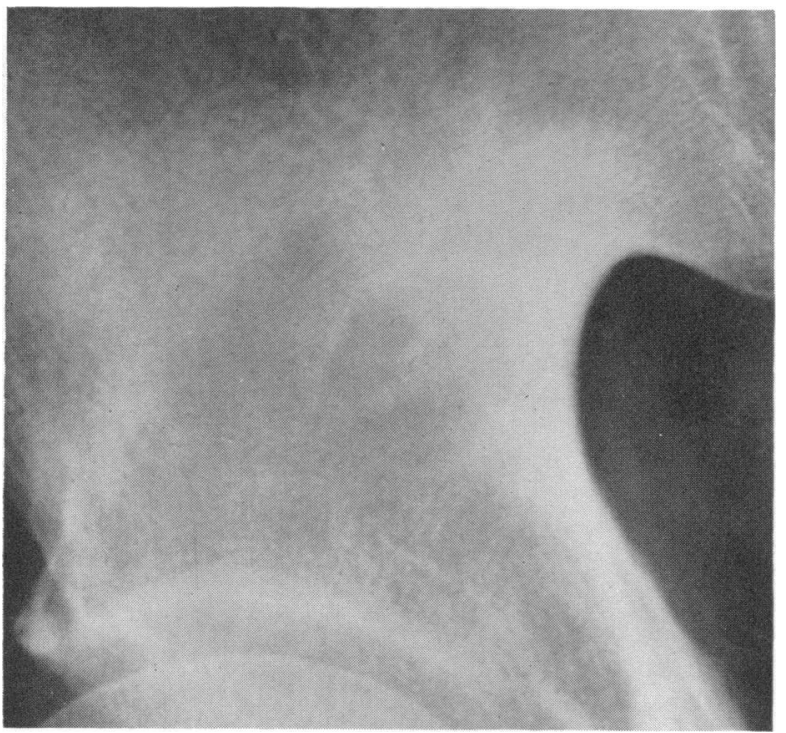

FIG. 5.-Radiograph of lesion in right ilium near pelvic brim.

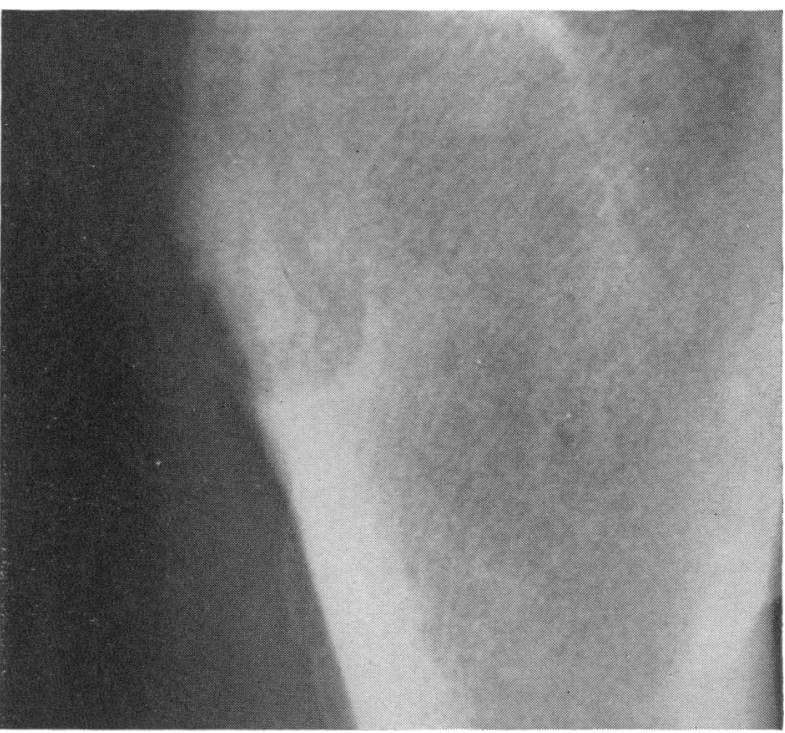

FiG. 6.--Radiograph of lesion below right greater trochanter.

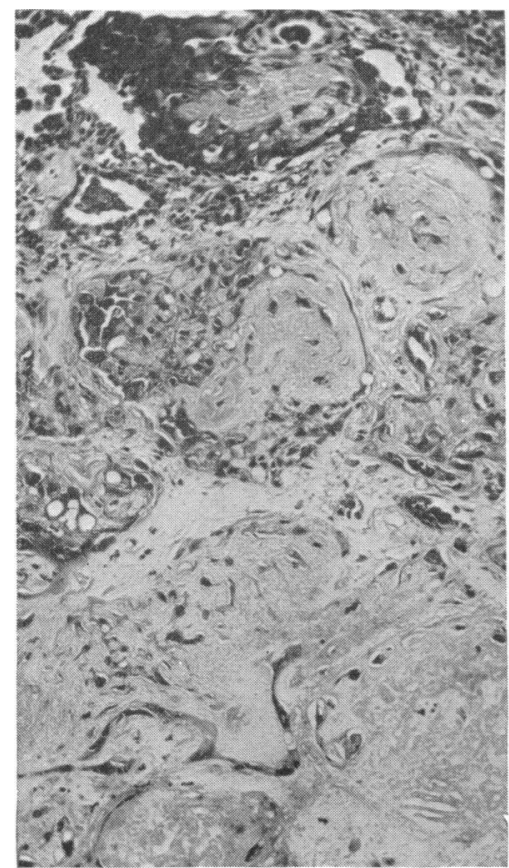

FIG. 7

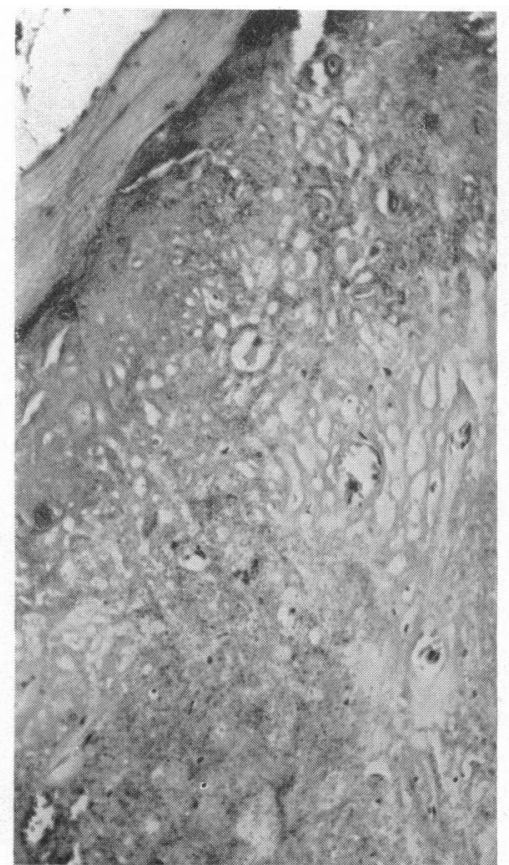

Fig. 8

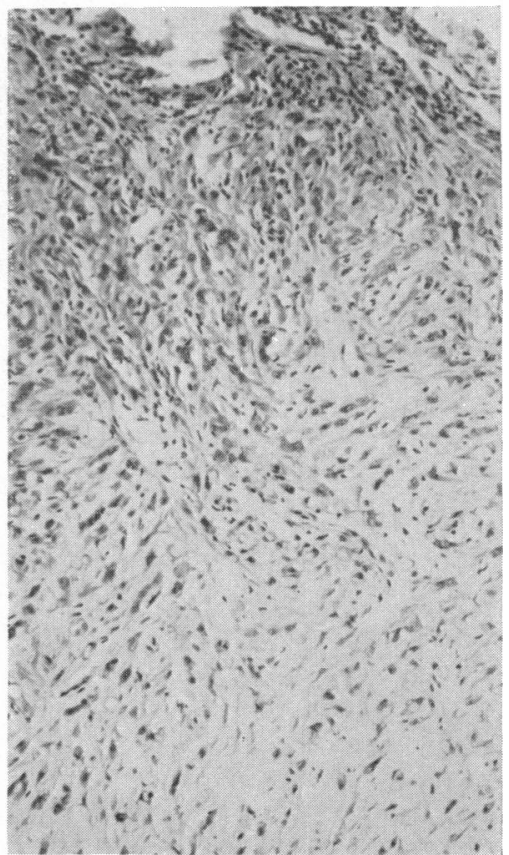

FIG. 9

Figs. 7, 8, and 9.- Sections of the lung, femur, and rib lesions respectively. The peripheries of the lesions are shown at the top, the inner parts below. (Haematoxylin and eosin. $\times 96$.)

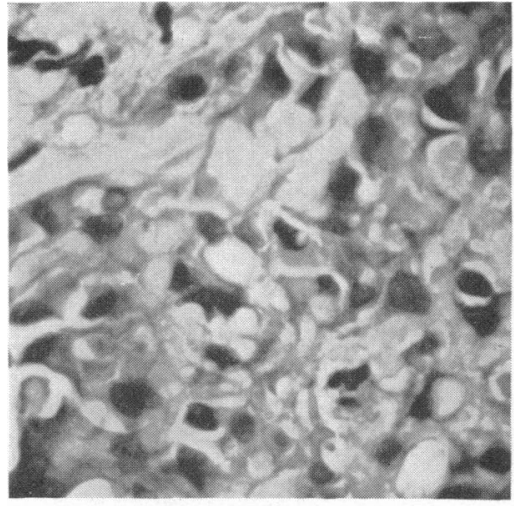

FIG. 10

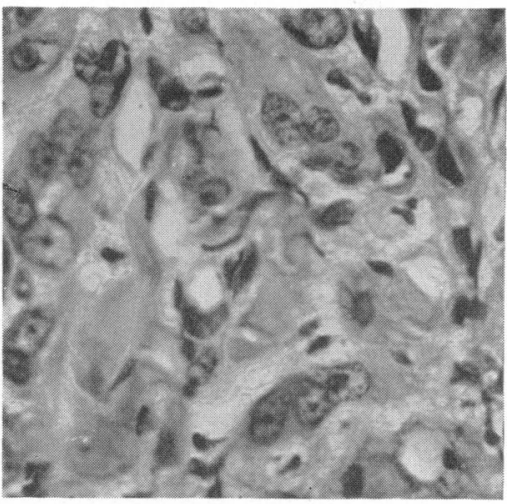

FIG. 11

FIGS. 10 and 11.-Cell detail from lesions in lung and rib respectively. (Haematoxylin and eosin. $\times 440$.) 
is more calcification than in 1956 , and more in the lower half of the lungs than in the upper. The calcification is at the periphery of the shadows, giving a rim of denser opacity about $1 \mathrm{~mm}$. thick: sometimes part of this peripheral annular shadow is enlarged to a thickness of about $3 \mathrm{~mm}$. (See Figs. 2 and 4.)

The bone picture has also changed. The translucent area below the right iliac crest has decreased in size from 15 to $9 \mathrm{~mm}$. across, and the central opacity has increased from 2 to $6 \mathrm{~mm}$. The other iliac lesion and the sacral lesion are less "punched out" in appearance, the difference between the osteolytic area and the surrounding sclerosis being less marked. The sclerosis of the body of the third lumbar vertebra is less marked, and the translucent area is difficult to see; there is, however, a little calcification in the centre and at the lower border of the lesion. There has been considerable reactive ossification at the biopsy site in the right femur.

The solitary lesion below the left diaphragm has changed very little.

\section{Histology}

In 1956 a small nodule was removed from the tip of the lingula. In 1957 a second biopsy was done, of the cystic area in the right greater trochanter. In 1959 a third biopsy was performed, on the lesion in the right second rib.

Biopsy of Lingula.-This nodule measured $4 \mathrm{~mm}$. across on the pleural surface and was about $2 \mathrm{~mm}$. deep. The pleural surface of the lesion is smooth, and the deep surface seems to correspond with the outlines of alveoli. The nodule is composed of faintly eosinophilic material, shown by van Gieson's stain to be composed of a network of collagen fibres. This material has filled the alveoli, the walls of which can be shown by appropriate stains to be preserved in the outer part of the lesion, though destroyed in the centre. The centre contains hardly any cells, but the periphery contains small numbers of cells. Most of these cells are polygonal or elongated, with irregular slender processes and rounded or oval nuclei showing a fine chromatin network and one or two small nucleoli. The cells are often arranged in a roughly circular fashion corresponding with the persistent alveolar structures. In some places on the extreme edge of the lesion there are aggregates of cells arranged round clumps of material which stains like fibrin. There are also a few lymphocytes here and there in the outer part of the nodule, but no neutrophils or eosinophils. Apart from the collagen no anisotropic material has been seen, nor has calcification, nor any sign of parasitic disease such as fragments of ova. (See Figs. 7 and 10.)

Biopsy of Right Greater Trochanter.-At operation " there was a ring of dense white bone with a soft centre in which lay a creamy white mass similar in size and shape to a rice grain." The sections show lamellar bone, with fatty marrow, surrounding a central mass which shows some granular calcification and is composed of almost acellular fibrous tissue, the fibres forming an irregular network in the meshes of which lie the few cells that are present. (See Fig. 8.) These cells are degenerate and are visible only by reason of their slender dark pyknotic nuclei. The bone is unusual only in being somewhat more cellular than skeletal bone usually is.

Biopsy of Right Second Rib.-A short piece of the right second rib was removed, including a $1-\mathrm{cm}$. mass of soft tissue lying in a concavity in the inferior border of the rib. Microscopically it is seen to be composed of a kind of fibrous tissue, peripherally fairly cellular but with little collagen fibre, centrally much less cellular and more collagenous, the fibres again forming a network resembling that seen in the lesions from lung and femur. The cells resemble those seen in the lung lesion, apart from being much more numerous. Vessels, mitotic figures, and inflammatory cells are all very scanty. This nodule is not calcified. (See Figs. 9 and 11.)

\section{Treatment and Progress}

In 1956, a great range of possible diagnoses having been considered, it was decided that pulmonary tuberculosis alone could not be definitely excluded. The patient was therefore given para-aminosalicylic acid and isoniazid daily for six weeks. This made no difference to his already excellent health or to the shadows in his lungs.

Since then he has remained perfectly well apart from occasional cough and sputum. He has been quite unconcerned about all the investigations. $\mathrm{He}$ is at present doing outdoor manual work, and can climb four flights of stairs without getting more than slightly breathless. His weight has been stationary. The E.S.R. has varied from 5 to 16 $\mathrm{mm}$. an hour, and the eosinophil count has never been significantly raised (maximum 636/c.mm.).

No treatment has been given since the brief antituberculosis course in 1956.

\section{Discussion}

The lesions in the lungs and bones have been observed during the same period of several years in the life of this patient, and during that period have shown somewhat similar radiographic appearances in that in both sites the lesions are round, are roughly the same size, have a peripheral rim of denser opacity, and, in some cases, an internal or central opacity. Moreover, in both lung and bone the lesions have slowly changed radiographically over the four years, gaining greater density.

There are also histological similarities between the two sets of lesions. In both we find cells of similar appearance producing masses of fibrous tissue, more cellular peripherally and less cellular centrally, and showing similar patterns of the component fibres. The rib lesion is the most active of the three lesions so far seen, though its centre is notably less cellular than its periphery. The lung lesion is much less cellular than the rib lesion; none the less, there appears to be continuing exudation at one or two points on the periphery, and there are a few lymphocytes round about. The lesion from the femur has so few cells that it might be thought to be "burnt out." The rib lesion could therefore be regarded as early, the femur lesion as late, and the lung lesion as intermediate.

There are, however, certain discrepancies between the lesions in the two sites. The proliferating cells have caused total erosion of bone but only partial destruction of lung, perhaps because space for cell multiplication can be found in the lung alveoli but not in solid cortical bone. Then, too, the lesions are much more numerous in the lungs than in the bones. Again, although both lung and bone lesions have the same rim of denser opacity, only a few of the lung lesions show radiographically a central opacity such as is seen in all the bone lesions except that in the rib.

However, because of the radiographic and histological resemblances between lung and bone lesions, and because of their synchronism, we propose as a working hypothesis that they are parts of a single disease.

The nature of the disease is a more difficult problem. Certain conditions can be excluded on histological grounds-namely, tuberculosis, sarcoidosis, rheumatoid lung, eosinophilic granuloma, the pneumoconioses, parasitic and fungal diseases such as histoplasmosis and schistosomiasis, and disseminated malignant neoplasm. The lung lesion bears no resemblance histologically 
to microlithiasis alveolarum or pulmonary alveolar proteinosis (Sharp and Danino, 1953 ; Finkbiner et al., 1957 ; Payseur et al., 1958 ; Rosen et al., 1958).

It seems to us that this disease must be either inflammatory or neoplastic or metabolic. As has been said, malignant neoplasm can be excluded. Benign neoplasm does not seem appropriate to the evolution of the lesions, which appear to show early activity and gradual quiescence. This points to an inflammation rather than a neoplasm, as does the presence of fibrin at the edge of the lung lesion.

The inflammatory agent, however, is clearly very mild. Clearly also it is neither readily eliminated nor readily neutralized by the tissue reaction which produces the lesions. The impression received is that the agent acts first on a given focus and stimulates the centrifugal proliferation of fibroblast-like cells, each of which produces a small amount of collagen and then degenerates, leaving a meshwork of collagen with hardly any cells. The whole process seems to start at the centre, to spread from the centre, and to die away from the centre. Calcification presumably occurs as a late sequel to the fibrosis, as is common enough.

The cells in the lesion are obviously capable of producing fibrous tissue, and in that sense may be called fibroblasts or fibrocytes. But in some places in the lung lesion some of the cells look like alveolar histiocytes, which indeed is the probable nature of the cells aggregated round the fibrin. Cells of similar appearance can also be found in the rib lesion. The cells may perhaps be histiocytes rather than fibrocytes, and the condition may be metabolic rather than inflammatory. It might be a storage disease such as eosinophilic granuloma (though the histology will not fit that particular condition).

One thinks also of the patient's years of work as a dustman and the possibility of his having inhaled some irritant substance. However, he did not start this work until 1950 and changes were already visible in the mass $x$-ray film of 1949.

As the lesions now seem to be quiescent, and as the patient's lung function is virtually unimpaired, it seems quite possible that his disease, whatever it is, will cause him no further trouble.

The lesion thought to lie in the mesentery is probably part of the same disease. The fact that, unlike the lung and bone lesions, it has not changed radiographically over the four years may be because when first seen it was already at a late stage of development and consequently nearly static. Its existence reminds us that the disease may be more generalized than we now realize.

For the present, however, with the reservation just mentioned, we suggest that this puzzling condition might be provisionally labelled "focal skeleto-pulmonary sclerosis."

We wish to thank Dr. Joyce A. Keeping, consultant physician in charge of the Paddington and Kensington Chest Clinic, for permission to publish this case. We are obliged to Dr. A. D. Morgan for sections of the lingular biopsy, to Dr. H. A. Sissons for sections of the femur biopsy, and to Dr. F. Campbell Golding for the 1956 radiographs of the pelvis and femur. We are grateful to them and to Professor Gough, Professor Newcomb, Professor Symmers, Dr. Lynne Reid and Dr. George Simion for helpful advice on the histology and on the radiographs.
REFERENCES

Finkbiner, R. B., Decker, J. P., and Cooper, D. A. (1957). Amer. Rev. Tuberc. 75, 122.

Payseur, Coyt R., Konwaler, B. E., and Hyde, Leroy (1958). Ibid., 78, 906.

Rosen, S. H., Castleman, B., and Liebow, A. A. (1958). New Engl. J. Med., 258, 1123.

Sharp, Mary E., and Danino, E. A. (1953). J. Path. Bact., 65, 389.

\section{OCCLUSIVE DISEASE OF VISCERAL ARTERIES \\ INTERNATIONAL CARDIOVASCULAR SOCIETY'S SYMPOSIUM}

\section{[By a Special Correspondent]}

The Fifth Congress of the International Cardiovascular Society was held in Dublin from September 7 to 9 in conjunction with the 19th Congress of the International Society of Surgery. Professor CHARLES G. RoB (Rochester, New York) gave as his presidential address the René Leriche Memorial Lecture, his subject being "Indications for Operation in Occlusive Disease of the Visceral Arteries." This was followed by a symposium on the surgical management of occlusive disease of the visceral arteries. The remarks of Professor Rob and other speakers are summarized below.

\section{Atherosclerosis of Cerebral Arteries}

Discussing the indications for surgery in atherosclerosis of the cerebral arteries, Professor Rob, with experience of 431 cases, 294 of which had been operated upon, classified his patients clinically into two groups-cases of transient or incipient stroke and cases of progressive or completed strokes. It was important to realize the significance of the transient or incipient stroke, since $60 \%$ of patients had a minor attack or attacks, perhaps over a period of weeks or months, before the progressive and complete episode of cerebral ischaemia developed.

Atherosclerotic stenosis or narrowing of the internal carotid artery at its origin was the usual pathological background of the transient or incipient group, while in the progressive or completed type thrombosis supervening on the stenosis was the common finding. However, the condition of the vertebral arteries should not be overlooked, and, as Dr. M. E. DEBAKEY (Houston) emphasized, arteriography, which was always essential, should aim to show at least three or perhaps all of the four main arteries to the brain. In some cases stenosis of the internal carotid might be present without symptoms, the other arteries usually being healthy. In fact, it was possible for symptoms to be absent even if only one vertebral artery was free of disease. Professor RoB pointed out that in diagnosis a systolic bruit over the carotid bifurcation, present in $45 \%$ of all cases with an internal-carotid-artery stenosis, was a useful sign, and doctors in practice should be encouraged to use the stethoscope in screening all possible cases. It was disappointing that the internist was slow to abandon his conservative approach to this vascular catastrophe: he should recognize that cerebral arteriography was a safe and simple procedure and that it alone could give precise information regarding the pathological background of the cerebral ischaemic symptoms and whether or not surgical treatment was advisable or feasible.

Professor Rob went on to say that surgery should be looked upon as a prophylactic procedure to prevent further attacks and the development of complete thrombos:s. Operation should take place without delay once the diagnosis was made, as with the onset of complete 